\title{
Género y Autonomía en la Adolescencia: Las diferencias entre chicos y chicas aumentan a los 16 años
}

\author{
Manuela Fleming
}

Dpto. de Ciencias del Comportamiento de la ICBAS,

Universidad de Porto

\section{Portugal}

manuelafleming@iol.pt 


\section{Resumen}

Introducción. La autonomía es un aspecto de gran importancia en el desarrollo de los adolescentes. Su logro es un mediador en la transición a la adultez. Implica varios parámetros psicológicos, entre los cuales se encuentran el deseo, el conflicto con los padres y el logro real de tal autonomía.

Método. Se ha investigado la percepción de la autonomía de adolescentes varones y mujeres que formaban una muestra considerable de adolescentes de 12 a 17 años $(\mathrm{n}=934)$. Fueron seleccionados de entre un grupo de 6.829 estudiantes de Educación Secundaria. Se registraron las respuestas de tres grupos de edad para examinar la frecuencia de respuestas afirmativas a un cuestionario de 11 ítems. Para cada ítem se evaluó el deseo de autonomía, el logro real de dicha autonomía y la desobediencia a los padres.

Resultados. De los 12-15 años de edad, las respuestas de los adolescentes varones y mujeres eran comparables en cuanto a la autonomía. A partir de los 16 años se encontraron diferencias importantes entre los adolescentes varones y mujeres: los chicos informaron de una frecuencia creciente de la consecuención real de autonomía, mientras las chicas no informaron de mucho avance. Los análisis estadísticos arrojaron que el mayor nivel de autonomía que mostraban los chicos adolescentes más mayores estaba asociado a una mayor frecuencia de desobediencia a los padres.

Discusión. Concluimos que: (i) existen diferencias importantes en cuanto a la autonomía de adolescentes asociadas al género; (ii) estas diferencias empiezan a aparecer a las edades de 16-17 años; (iii) en la adolescencia posterior, los chicos muestran un mayor nivel de logro de autonomía que las chicas, y éste se asocia con una mayor incidencia de desobediencia a los padres entre los chicos.

Palabras clave: Adolescencia, Psicología, Autonomía, Cuestionario, Comportamiento. 


\section{Introducción}

Steinberg y Silverberg (1986) documentaron la complejidad y el papel central que ocupa la autonomía durante la adolescencia, y esto ha dado lugar a muchas investigaciones sobre los cambios psicológicos de los adolescentes conforme van llegando a la adultez (p.ej., Allen et al., 2002; Beyers y Goossen, 1999; Fleming, 1992; Noom et al, 2001; Ryan y Lynch, 1989; Steinberg, 1990; Schmitz y Baer, 2001). En la actualidad existe consenso de que el nivel de logro de comportamientos autónomos es un modulador clave del proceso de transición de la adolescencia hacia la adultez (Benaches, 1981; Chou, 2000; Fleming y Aguiar, 1992; Frank et al., 2002; McElhaney y Allen, 2001; Noom et al., 1999). Por ejemplo, hay evidencia de asociación entre el fracaso al intentar independizarse, y un progreso deficiente en los comportamientos autónomos de los adolescentes (Chen, 1999; Cohen et al., 2003; Fleming, 1983; Moore, 1987; Pinheiro et al., 2001).

La investigación actual considera el papel de la edad y el género en la modulación de la autonomía adolescente. Se emplea aquí el término de autonomía adolescente en el mismo sentido que la definición propuesta por Steinberg (1999), es decir, la capacidad individual de pensar, sentir, tomar decisiones y actuar por uno mismo. El desarrollo de la independencia naturalmente representa un componente decisivo en el proceso de ser autónomo, pero la autonomía significa algo más que el simple comportarse con independencia. Se cree que la autonomía se desarrolla a través de relaciones en el seno de la familia, con los iguales y también con personas externas a la familia (Purdie et al., 2004). El conflicto familiar provocado por la autonomía de los adolescentes, al desobedecer las normas de sus padres, puede ser considerado un componente normal del desarrollo psicológico e implica cambios en las relaciones familiares (Steinberg, 1999).

Varias investigaciones han documentado que las puntuaciones de conducta autonóma aumentan gradualmente a lo largo de la adolescencia, un fenómeno que se refleja en las marcadas diferencias que con frecuencia se observan entre la autonomía de los adolescentes más jóvenes y la de los más mayores (Bartle et al., 1988; Dornbush et al., 2001). Los adolescentes más mayores logran mayor autonomía particularmente en su elección de amistades y de profesión, en el manejo de su propio dinero y en actividades físicas realizadas fuera del hogar familiar (Allen et al., 2002; Douvan y Adelson, 1966). También manifiestan mayor capacidad en la integración social (Greenberger, 1984) y en 
la participación en actividades orientadas hacia adultos o hacia los compañeros (Silverberg y Steinberg, 1987). El aumento en las puntuaciones de conductas autónomas en la adolescencia ha sido relacionado con un descenso en la influencia de los padres (Smith y Crawford, 1986) y con un aumento gradual de la afiliación con los compañeros (Bergen et al., 2003; Peppitone, 1980; Pipp et al., 1985;).

Recientemente, Stewart y McDermott (2004) han examinado y subrayado la importancia de comprender el significado del género en los procesos psicológicos. Algunas investigaciones recientes se han dedicado a la identificación de diferencias de género en la psicología del adolescente. Por ejemplo, a las chicas de familias donde la madre mantiene actitudes tradicionales sobre el papel del género se les concedían menos oportunidades para la autonomía (Bumpus et al., 2001); llegar a ser autónoma era una experiencia más estresante para las chicas que para los chicos (Beyers y Goossen, 1999; Lamborn y Steinberg, 1993); las chicas tenían puntuaciones más altas para identidad y puntuaciones más bajas para intimidad y explicaban sus decisiones más que los chicos (Lacombe y Gay, 1998); las chicas que salían con chicos mostraban el conflicto más intenso con los padres (Dowdy y Kliewer, 1998). La influencia de los compañeros en la conducta en la adolescencia temprana depende del género del adolescente y éste último modula la identificación con el grupo y el comportamiento delincuente (Kiesner et al., 2002 y 2003).

El género modula la autoimagen del adolescente: por ejemplo, la insatisfacción con el cuerpo y las dietas son dos veces más frecuentes en las chicas (Borresen y Rosenvinge, 2003). Cuando se compara la autoevaluación del logro de la autonomía del adolescente y el adulto joven entre los dos sexos, se encuentra una conexión más estrecha entre los dos momentos para las chicas; es decir, una autoevaluación negativa en la adolescencia estaba más a menudo asociada a una autoevaluación negativa en la adultez en las chicas que en los chicos (Verhofstadt-Deneve et al., 2003). Está claro que las diferencias de género están presentes en la psicopatología, con una incidencia más alta de morbilidad en las chicas que en los chicos (Arnold, 2003; Joseph et al., 2003; Sloan y Kornstein, 2003;). Por ejemplo, la frecuencia de abuso sexual que sufren las chicas adolescentes es más alta que la de chicos y éstas tienen mayor riesgo de desarrollar una patología y de intento de suicidio (Bergen et al., 2003). Asimismo, el género influye en el índice de 
búsqueda de ayuda de los adolescentes para sus problemas emocionales (Pinto, 2004; Zwaanswijk et al., 2003).

El propósito del presente trabajo fue investigar las diferencias de género y los cambios relacionados con la edad durante la adolescencia (desde la pubertad hasta los 17 años) en cuanto a la autonomía en tres dimensiones psicológicas: el deseo de autonomía, el logro real de autonomía y la desobediencia a los padres. Hasta ahora no se habían estudiado estas dimensiones simultáneamente. El modelo teórico que orienta esta investigación es psicodinámico, centrándose en las teorías de la separación-individuación y de la formación de la identidad (Blos, 1967; Josselson, 1980; Mahler et al, 1975); éstas conciben la autonomía como una tarea crítica del desarrollo, la cual prepara al adolescente para una transición gradual a los roles del adulto. El ajuste a la adultez depende de la medida en que el adolescente logra con éxito la separación y la desconexión con sus padres (Silverberg y Steinberg, 1987). Estos modelos relacionan de forma indirecta la autonomía con la individuación y con el apego emocional, dos procesos psicológicos que interfieren en el dilema psicológico del adolescente: el deseo y el miedo de separarse de los padres.

Los objetivos de la investigación se realizaron comparando las percepciones de autonomía entre cuatro grupos de adolescentes de distinta edad. En lugar de utilizar un concepto de autonomía definido de antemano por la investigadora, se decidió usar un cuestionario basado en la autonomía tal como la definen los adolescentes mismos. Este cuestionario fue validado y adoptado en una investigación anterior (Fleming, 2005). Nuestros datos documentan la importancia relativa de once comportamientos de autonomía en una población amplia, entre los 12 y los 17 años de edad. Por otra parte, los resultados identifican similitudes y diferencias entre los chicos y las chicas en cuanto a cómo se percibe y se vive la autonomía a lo largo de la adolescencia.

\section{Método}

\section{Participantes}

La muestra está formada por estudiantes de Educación Secundaria, seleccionados de entre una población de 6.829 chicos de 12 a 17 años de edad. Se seleccionó una muestra de 934 individuos según criterios de representación proporcional de edad. El 52\% fueron chicos y el 48\% chicas, entre los 12 y 17 años (media de edad: 13,1 años). La gran 
mayoría de estos adolescentes (97\%) vivían con ambos padres biológicos; sólo el 3\% vivían en una familia monoparental. Había un amplio espectro de estatus social y económico entre las familias de los adolescentes.

\section{Materiales y Procedimiento}

Identificación de los items principales de conductas de autonomía en los adolescentes. Para empezar, se diseñó un cuestionario con el objeto de establecer los ítems que los adolescentes consideraron indicativos de conducta autónoma. Las preguntas tenían que ver con: qué era para ellos la autonomía, qué era para ellos dejar de ser niño, cuáles eran las expresiones de autonomía en su vida diaria y qué comportamientos representaban para ellos la autonomía. Se realizó un análisis de contenido de las respuestas al cuestionario de una muestra de adolescentes $(n=40)$ con una distribución de edades parecida a la población más numerosa de adolescentes que participaron en la segunda fase del trabajo. Esto permitió la identificación de 11 ítems de autonomía que con más frecuencia citaron los adolescentes.

Estos 11 ítems se muestran en la Tabla 1 y fueron adoptados para construir un cuestionario de comportamientos de autonomía de adolescentes que luego se utilizó como autoinforme de autonomía; la consistencia interna de la escala fue 0,74 según el alfa de Cronbach. Un total de 934 adolescentes rellenaron el cuestionario con respuestas de sí/no para tres aspectos de cada ítem: deseo, logro y desobediencia a los padres.

\section{Tabla 1: 11 Ítems Seleccionados}

1. Decorar mi habitación como yo quiera.

2. Vestirme y llevar el pelo como me guste.

3. Tener mi propio dinero y gastarlo sin supervisión.

4. Salir de noche.

5. Salir sin tener que decir adónde voy.

6. Entrar y salir de casa como yo quiera.

7. Salir los fines de semana.

8. No pasar los días festivos con la familia.

9. Tener novia o novio.

10. Resolver mis propios problemas sin la ayuda de mis padres.

11. Tener mis propias ideas sobre política, religión y educación. 
Grupos de edad. La muestra de 934 adolescentes se dividió en 3 grupos de las siguientes edades: 12-13 $(\mathrm{n}=549), 14-15(\mathrm{n}=261)$, y 16-17 $(\mathrm{n}=124)$. Se comparó la incidencia de respuestas afirmativas a los 3 parámetros (deseo de autonomía, logro de autonomía y desobediencia a los padres), para cada uno de los ítems entre los chicos y las chicas de cada uno de los grupos de edad.

\section{Análisis Estadístico}

Se realizó la comparación estadística para cada género y grupo de edad, para los 11 ítems de conducta autónoma. Analizamos las frecuencias de los datos y las diferencias estadísticamente significativas, tomando como variables independientes el género y la edad. En cuanto a los parámetros de género y de edad, comparamos las diferencias entre las proporciones utilizando la prueba de Chi-cuadrado y la prueba para tendencia lineal (Paquete BMDP), con la edad como variable ordinal.

\section{Resultados}

\section{Análisis preliminar}

Se pidió a los adolescentes que registraran una respuesta de sí/no para cada uno de los 11 ítems de conducta autónoma (enumerados en la Tabla 1), ante 3 preguntas: (i) ¿Se deseaba esta conducta? (ii) ¿Se había conseguido? (iii) ¿Era dicho comportamiento una causa para desobedecer el consejo de los padres?

La Figura 1 muestra los porcentajes de adolescentes varones y mujeres que respondieron sí ante cada uno de los 11 ítems enumerados en la Tabla 1. Esta figura está organizada en 3 pares de gráficos; en la izquierda están los resultados de los varones y en la derecha están los de las chicas; los 3 grupos de edad (12-13, 14-15, y 16-17) están indicados entre cada par de gráficos. En cada gráfico, se trazan tres líneas: la línea superior (cuadrados) hace referencia al porcentaje de respuestas afirmativas a cada uno de los 11 ítems (Tabla 1) en cuanto a deseo de autonomía; la línea media (triángulos) indica las respuestas afirmativas para la consecución de autonomía en los 11 ítems, y la línea de media/inferior (puntos) muestra las respuestas afirmativas sobre la desobediencia a los padres para cada uno de los 11 ítems. 
Figura 1. Comparación entre los 3 grupos de edad (centro) de adolescentes varones (izquierda) y de mujeres (derecha) en cuanto al porcentaje de chicos que expresaron deseo (línea superior, cuadrados), consiguieron (línea media, triángulos) o desobedecieron a sus padres (línea inferior, círculos) respecto a 11 comportamientos de autonomía, enumerados en la Tabla 1 y mostrados aquí en el mismo orden numérico.

Chicos

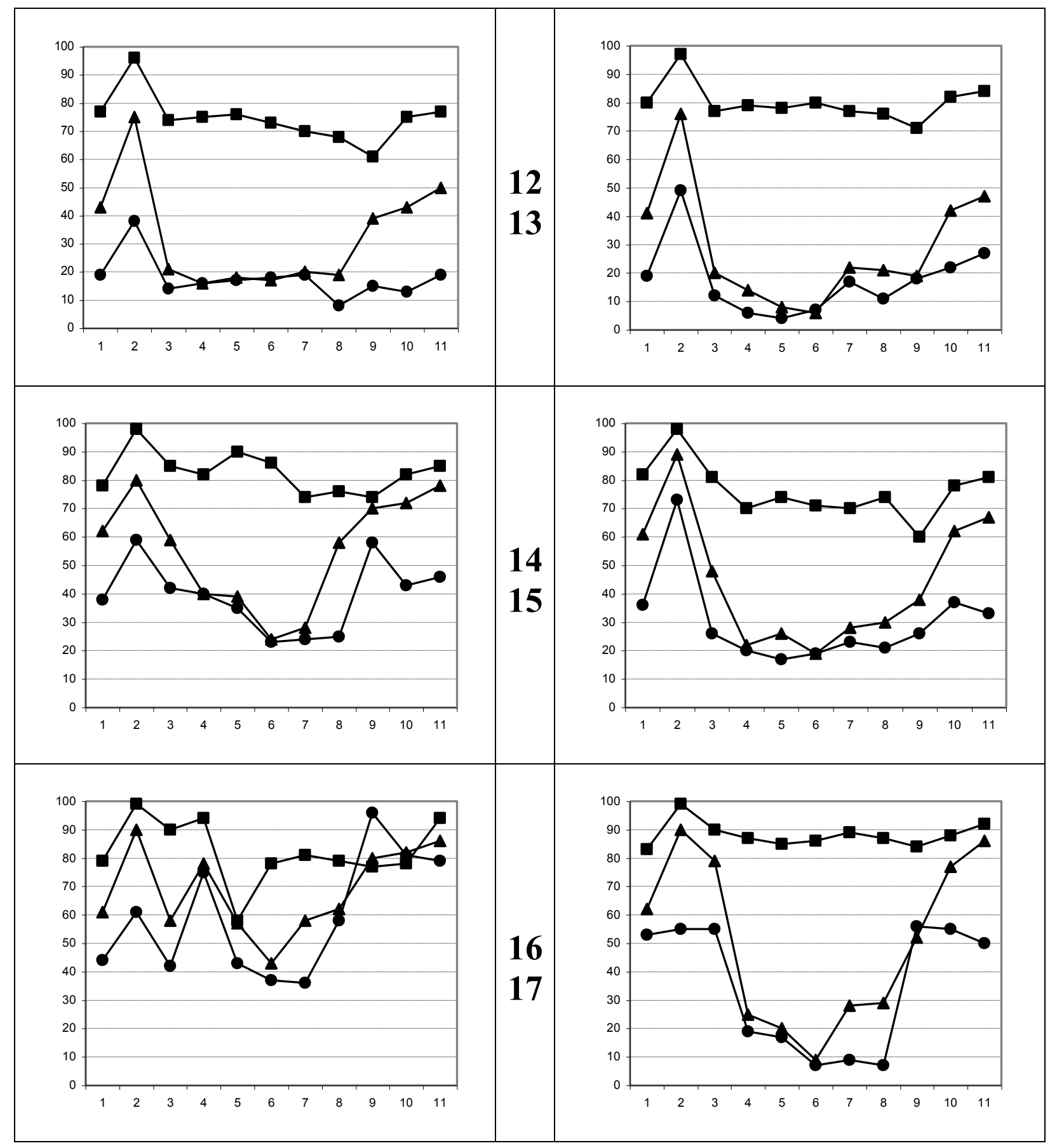


El análisis de los datos representados en la Figura 1 deja ver que: (i) una mayoría de adolescentes desean autonomía, particularmente cuando llegan a la edad de 14 años; (ii) la frecuencia de deseo de autonomía es mayor que la frecuencia de consecución real, especialmente en las chicas; (iii) las poblaciones masculina y femenina se diferencian a partir de los 16 años en adelante; (iv) la consecución de autonomía está asociada a la desobediencia a los padres (en la Figura 1, las líneas para logro de autonomía y desobediencia a los padres se van acercando, sobre todo para los chicos).

La frecuencia de deseo de comportamientos autónomos es el único parámetro que no distingue claramente entre los adolescentes chicos y chicas: la mayoría de los 11 ítems de comportamiento eran deseados por un $70 \%$ ó más de los chicos y chicas; y por los adolescentes más jóvenes (12-13 años) y los más mayores (16-17 años). Para establecer diferencias estadísticas, se realizó una prueba de Chi-cuadrado, comparando chicos y chicas (Tabla 2).

Tabla 2. Asociación entre deseo de autonomía y género. Los valores significativos indican diferencias en cuanto a deseo para el ítem correspondiente entre chicos y chicas.

\section{Ítems de autonomía}

$X 2$

gl

p

1. Decoración

2. Ropa

0.131

3. Dinero

4.609

1.192

4. Salir de noche

5.245

5. Salir sin decir adónde

7.460

6. Salir sin horario

6.465

7. Salir los fines de semana

0.0001

8. Festivos separado de la familia

0.015

9. Tener novio/novia

2.138

10. Decidir sin consejos

0.981

11. Ideología propia

0.0001

$0.716 n s$

$0.032 *$

$0.275 n s$

$0.022 *$

1

$0.006^{* *}$

1

$0.011^{*}$

1

$0.979 n s$

$0.901 n s$

1

$0.144 n s$

ns, no significativo; ${ }^{*}$ significativo $(\mathrm{p}<.05) ; * *$ muy significativo $(\mathrm{p}<.01) ; * * *$

$0.322 n s$ altamente significativo $(\mathrm{p}<.001)$; gl, grados de libertad.

La prueba indica que la interacción de género/ítems de autonomía no es significativa para 7 de los 11 ítems. Las diferencias estadísticamente significativas se limitaban a que: (i) las chicas deseaban elegir su propia ropa y peinado (ítem 2) más que los chicos y (ii) 
los chicos deseaban poder salir sin control de los padres (ítems 4, 5 y 6) más que las chicas. Las Tablas 3 y 4 muestran los datos sobre diferencias de edad, tomando de forma separada los chicos y las chicas.

Tabla 3. Asociación entre deseo de la autonomía y edad en adolescentes varones. Los valores significativos indican que los adolescentes más jóvenes (12-13 años) y los adolescentes más mayores (16-17 años) son distintos en cuanto a su deseo para dicho ítem.

\begin{tabular}{llll}
\hline \multicolumn{1}{c}{ Ítems de autonomía } & X2 & $\boldsymbol{g l}$ & $\boldsymbol{p}$ \\
\hline & & & \\
\hline 1. Decoración & & & \\
2. Ropa & 0.496 & 3 & $0.919 n s$ \\
3. Dinero & $6.116(\mathrm{a})$ & 1 & $0.013 *$ \\
4. Salir de noche & $11.749(\mathrm{a})$ & 1 & $0.003^{* *}$ \\
5. Salir sin decir a dónde & $10.642(\mathrm{a})$ & 1 & $0.001^{* *}$ \\
6. Salir sin horario & 17.575 & 3 & $0.0001^{* * *}$ \\
7. Salir los fines de semana & 1.540 & 3 & $0.673 n s$ \\
8. Festivos separado de la familia & 4.852 & 3 & $0.183 n s$ \\
9. Tener novia & 13.819 (a) & 1 & $0.0001^{* * *}$ \\
10. Decidir sin consejos & 7.191 & 3 & $0.066 n s$ \\
11. Ideología propia & $7.517(\mathrm{a})$ & 1 & $0.006^{* *}$ \\
\hline
\end{tabular}

(a) Prueba para Tendencia Lineal; $n s$, no significativo; ${ }^{*}$ significativo $(\mathrm{p}<.05)$; ${ }^{* *}$ muy significativo $(\mathrm{p}<.01) ; * * *$ altamente significativo $(\mathrm{p}<.001)$; gl, grados de libertad.

Tabla 4. Asociación entre logro de autonomía y edad (adolescentes jóvenes, de 12-13 años, $y$ adolescentes más mayores, 16-17 años) en los chicos. Los valores significativos indican que los adolescentes más jóvenes y más mayores son distintos respecto a su logro del ítem.

\section{Ítems de autonomía}

$x 2$

gl

p

1. Decoración

2. Ropa

3. Dinero

4. Salir de noche

5. Salir sin decir a dónde

6. Salir sin horario

7. Salir los fines de semana

8. Festivos separado de la familia

9. Tener novia

10. Decidir sin consejos

11. Ideología propia

\begin{tabular}{|c|c|c|}
\hline 10.278 (a) & 1 & $0.001 * *$ \\
\hline 19.537 (a) & 1 & $0.0001 * * *$ \\
\hline 82.947 (a) & 1 & $0.0001 * * *$ \\
\hline $125.340(\mathrm{a})$ & 1 & $0.0001 * * *$ \\
\hline $52.876(\mathrm{a})$ & 1 & $0.0001 * * *$ \\
\hline 19.400 (a) & 1 & $0.0001 * * *$ \\
\hline 31.829 (a) & 1 & $0.0001 * * *$ \\
\hline 57.136 (a) & 1 & $0.0001 * * *$ \\
\hline 57.458 (a) & 1 & $0.0001 * * *$ \\
\hline 54.171 (a) & 1 & $0.0001 * * *$ \\
\hline 57.986 (a) & 1 & $0.0001 * * *$ \\
\hline
\end{tabular}


(a) Prueba para Tendencia Lineal; $n s$, no significativo; * significativo $(\mathrm{p}<.05)$; ** muy significativo $(\mathrm{p}<.01) ; * * *$ altamente significativo $(\mathrm{p}<.001)$; gl, grados de libertad.

Estos datos indican que, a medida que se hacen mayores, hay varios comportamientos que son cada vez más deseados por ambos sexos: gastar dinero (ítem 3), resolver los problemas propios (ítem 10) y tener convicciones propias (ítem 11).

Se observa un gran vacío entre las líneas de deseo de autonomía y de logro de dicha autonomía en todos los gráficos de la Figura 1, con la excepción de las líneas referentes a los chicos después de cumplir los 16 años. Esto indica que, para la mayoría de adolescentes, hay una disociación entre el deseo de autonomía y su logro. En las chicas, incluso entre las más mayores, la autonomía se limita a unos pocos ítems, a saber, los relacionados con la autonomía emocional (ítem 9: tener novio), o la autonomía cognitiva (item 10: resolver problemas sin ayuda de los padres; ítem 11: ideología propia).

En realidad, respecto a la dimensión deseo, los datos dejan ver diferencias de género solamente en 4 ítems, mientras que para la dimension logro, los datos muestran que los chicos y las chicas se diferencian en 8 ítems: las chicas consiguen autonomía en la decoración de su habitación (ítem 1), y en vestirse (ítem 2), con más frecuencia que los chicos; y los chicos consiguen autonomía en salir sin control de los padres (ítems 4, 5, 6, 7 y 8), y en tener pareja (ítem 9), con más frecuencia que las chicas, tal como se describe en la Tabla 5.

Tabla 5. Asociación entre consecución de autonomía y edad (adolescentes más jóvenes, 12-13 años, $y$ adolescentes más mayores, 16-17 años), en las chicas. Los valores significativos indican que las más jóvenes y las más mayores son distintas en cuanto al logro de dicho ítem.

\section{Ítems de autonomía}

1. Decoración

2. Ropa

3. Dinero

4. Salir de noche

5. Salir sin decir a dónde

6. Salir sin horario

7. Salir los fines de semana

8. Festivos separada de la familia
$X 2$

9.902 (a)

9.467 (a)

60.384 (a)

24.180 (a)

13.920 (a)

3.815

3.040

18.739 (a)

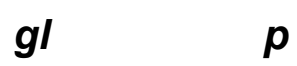

p

$0.001 * *$

$0.002 * *$

$0.0001 * * *$

$0.0001 * * *$

$0.0001 * * *$

$0.282 n s$

$0.385 n s$

$0.0001 * * *$ 
9. Tener novio

10. Decidir sin consejos

11. Ideología propia

76.028 (a)
45.439 (a)
48.387 (a)

$0.0001 * * *$

$0.0001 * * *$

$0.0001 * * *$

(a) Prueba para Tendencia Lineal; $n s$, no significativo; * significativo $(\mathrm{p}<.05) ; * *$ muy significativo $(\mathrm{p}<.01) ; * * *$ altamente significativo $(\mathrm{p}<.001)$; gl, grados de libertad.

Se encontraron diferencias importantes entre los chicos y las chicas cuando los adolescentes cumplían los 16 años. Esta diferencia de género se debe a un aumento brusco en la frecuencia de logro de autonomía de la que informaron los chicos de esta edad, un cambio que no mostraba un porcentaje comparable entre las chicas de la misma edad. Los análisis de los distintos ítems mostraron que las chicas no conseguían alcanzar una frecuencia alta de logro de conductas autónomas relacionada con la separación temporal de la familia (ítems 4-8: salir de noche, los fines de semana, los días festivos).

En cambio, los chicos registran un aumento significativo de su autonomía con la edad para todos los items (Tabla 6); mientras que para las chicas, hay dos ítems de comportamiento que no muestran un aumento significativo con la edad: el ítem 6 (salir los fines de semana) y el ítem 7 (pasar los días festivos lejos de la familia) (Tabla 7).

Tabla 6. Asociación entre capacidad de desobedecer y edad (adolescentes más jóvenes, 12-13 años, y adolescentes más mayores, 16-17 años) en los chicos. Los valores significativos indican que los más jóvenes y los más mayores son distintos en cuanto a su capacidad de desobedecer a sus padres para dicho ítem.

\section{Ítems de autonomía}

$x 2$

gl

$p$

1. Decoración

2. Ropa

3. Dinero

4. Salir de noche

5. Salir sin decir a dónde

6. Salir sin horario

7. Salir los fines de semana

8. Festivos separado de la familia

9. Tener novia

10. Decidir sin consejos

11. Ideología propia

\begin{tabular}{lll}
5.214 (a) & 1 & $0.022 n s$ \\
8.324 (a) & 1 & $0.003^{* *}$ \\
41.814 (a) & 1 & $0.0001^{* * *}$ \\
65.553 (a) & 1 & $0.0001^{* * *}$ \\
43.977 (a) & 1 & $0.0001^{* * *}$ \\
21.546 (a) & 1 & $0.0001^{* * *}$ \\
8.292 (a) & 1 & $0.004^{* *}$ \\
15.339 (a) & 1 & $0.0001^{* * *}$ \\
19.555 (a) & 1 & $0.0001^{* * *}$ \\
19.790 (a) & 1 & $0.0001^{* *}$ \\
13.112 (a) & 1 & $0.0001^{* * *}$ \\
\hline
\end{tabular}

(a) Prueba para Tendencia Lineal; $n s$, no significativo; $*$ significativo $(\mathrm{p}<.05) ; * *$ muy significativo $(\mathrm{p}<.01) ; * * *$ altamente significativo $(\mathrm{p}<.001)$; gl, grados de libertad. 
Tabla 7. Asociación entre capacidad de desobedecer y edad (adolescentes más jóvenes, 12-13 años, $y$ adolescentes más mayores, 16-17 años) en las chicas. Los valores significativos indican que las más jóvenes y las más mayores son distintas en cuanto a su capacidad de desobedecer a sus padres para dicho ítem.

\section{Ítems de autonomía}

1. Decoración

2. Ropa

3. Dinero

4. Salir de noche

5. Salir sin decir a dónde

6. Salir sin horario

7. Salir los fines de semana

8. Festivos separada de la familia

9. Tener novio

10. Decidir sin consejos

11. Ideología propia $x 2$

8.426

6.348

25.917 (a)

8.147 (a)

7.189 (a)

5.593

5.127

5.915

50.866 (a)

7.209 (a)

0.392

\section{gl}

p

(a) Prueba para Tendencia Lineal; $n s$, no significativo; * significativo ( $\mathrm{p}<.05) ; * *$ muy significativo $(\mathrm{p}<.01) ; * * *$ altamente significativo $(\mathrm{p}<.001)$; gl, grados de libertad.

Los datos en conjunto, según la Figura 1, sugieren que la diferencia de género más importante en cuanto a la autonomía adolescente es la capacidad de los chicos de 16 años de incrementar la frecuencia de acciones de desobediencia a los padres. Esta capacidad para el conflicto distingue claramente a los chicos y chicas de esta edad y en adelante. Para los chicos, el único ítem que seguía bajo en cuanto a la desobediencia a los padres (sobre $20 \%$ de respuestas afirmativas) fue el de pasar días festivos lejos de la familia. Por contra, para las chicas, 5 de los 11 ítems de autonomía eran puntuados constantemente por menos del $20 \%$ en cuanto a la desobediencia a los padres; estos ítems tenían que ver principalmente con situaciones de separación temporal de la familia.

El análisis estadístico muestra que los chicos son distintos a las chicas debido a una frecuencia más alta en la desobediencia a los padres, concretamente para los ítems 4,5 y 6 , que se relacionan con la evitación del control de los padres. En conjunto, teniendo en cuenta las diferencias en el logro de autonomía entre los chicos y las chicas expuestas anteriormente, es pertinente considerar que la mayor capacidad para desafiar la autoridad 
paterna expresada por los chicos está relacionada con el logro de niveles más altos de autonomía.

\section{Discusión}

El propósito de este trabajo fue investigar las diferencias de edad y de género en los adolescentes en cuanto a su forma de percibir la autonomía. Se llevó a cabo determinando la frecuencia con la que los adolescentes desean y logran la autonomía y la frecuencia con la que los comportamientos autónomos son considerados por los adolescentes como causa de conflicto con los padres. El análisis estadístico deja ver que el deseo de la autonomía, la consecución real de ésta y la desobediencia a los padres varían en función de la edad y el sexo de los adolescentes.

Los datos indican que existen solamente pequeñas diferencias entre los dos sexos en cuanto a su deseo de autonomía. Por contraste, hemos encontrado marcadas diferencias de género en cuanto al logro de la misma: la tendencia hacia la autonomía fue claramente más pronunciada en los chicos que en las chicas. Nuestro trabajo también deja ver que las diferencias de género más significativas ocurren hacia la mitad de la adolescencia, es decir, la distinción entre los chicos y las chicas se incrementa a los 16 años.

El análisis de contenidos, que llevó a la selección de 11 ítems de autonomía, y la proporción de adolescentes que a partir de los 12-13 años desean su consecución, apoyan la idea de que la autonomía en la adolescencia no es unidimensional, sino que se expresa en distintas dimensiones: aspectos actitudinales, emocionales y reguladores del comportamiento de los adolescentes, tal como han establecido investigadores anteriores (véase, por ejemplo, Noom et al, 2001; Ryan y Lynch, 1989; Steinberg, 1990; Steinberg y Silverberg, 1986).

Respecto a los cambios en autonomía según la edad, nuestros hallazgos respaldan los trabajos previos que han identificado un aumento en las puntuaciones de autonomía a lo largo de la adolescencia, reforzando así la autonomía como un valor clave entre las tareas evolutivas de la adolescencia en ambos sexos (Bartle et al. 1988; Bosma et al. 1996; Dornbush et al, 2001; Douvan y Adelson, 1966; Greenberger, 1984; Peppitone, 1980; Pipp et al., 1985). 
La importancia de comprender el significado del género en los procesos psicológicos, subrayada recientemente por Stewart y McDermott (2004), ha encontrado apoyo en este estudio. De hecho, el presente trabajo ha identificado importantes diferencias de género en varios parámetros de la autonomía de los adolescentes. El género modula la autoimagen: por un lado, la elección autónoma de vestido y peinado eran con más frecuencia citadas entre las chicas que entre los chicos, como encontraron también Borresen y Rosenvinge (2003), y, por otro lado, la actividad exploratoria fuera del entorno de la familia era con más frecuencia citada entre los chicos que entre las chicas.

El hallazgo principal de este trabajo es que, en lo referente a la autonomía, la diferenciación entre ambos sexos ocurre a los 16 años. De hecho, los chicos lograron la autonomía antes que las chicas, a pesar de que se detectaban niveles parecidos de frecuencia de deseo de autonomía en ambos sexos. Nuestros resultados indican que esta diferencia proviene, al menos en parte, de la mayor capacidad, expresada por los chicos, de desobedecer las reglas de los padres. Las chicas se limitaban a entrar en conflicto con los padres sólo por los ítems emocionales, como el de tener novio; la preocupación emocional de las chicas también ha sido señalada previamente (Taradesh et al., 2001).

Este trabajo confirma que la mayoría de los adolescentes, con independencia de su sexo, desea la autonomía. Este rasgo psicológico de los estudiantes de secundaria y residentes de barrios urbanizados aquí investigados ha sido también identificado en adolescentes de otros entornos culturales y sociales (Anderson y Anderson, 1986; Benaches, 1981; Konopka, 1983; Meyer, 1988; Steinberg y Silverberg, 1986; Williamson y Campbell, 1985). Evidentemente, hay que considerar que los rasgos específicos de la cultura portuguesa también han modulado nuestros datos. Nuestro cuestionario puede en estos momentos ser empleado en estudios comparativos acerca de la psicología de la adolescencia en distintos grupos geográficos, nacionales o étnicos.

Se observó aquí una disparidad entre el deseo y el logro real en la mayoría de los grupos de edad. Esto indica que los adolescentes tienen que enfrentarse a su deseo de autonomía, lo que es consecuente con el hallazgo de que el conflicto con los padres fomenta la autonomía, tal como se deduce de la asociación encontrada entre un aumento 
en la frecuencia de desobediencia a los padres y el logro de autonomía que citaron los chicos adolescentes de mayor edad.

Concluyendo, este trabajo establece que los 16 años constituyen un hito en la diferenciación entre sexos en la adolescencia respecto al logro de su autonomía. Este trabajo sugiere que el hecho de que los chicos consigan autonomía de forma más temprana no se debe a un nivel más alto de deseo de autonomía, sino a una capacidad anterior de expresar conflicto con los padres.

Nuestros hallazgos sugieren que las chicas valoran la autonomía y la agencia personal tanto como los chicos, pero éstas no demuestran la misma capacidad de luchar como los chicos, manteniendo más dependencia de las normas de los padres. También apoyan la idea de muchos expertos e investigadores de que los hombres valoran la autonomía más que las mujeres. Esto quizás se deba al hecho de haber tenido en cuenta sólo el logro y no el deseo de autonomía, una dimensión crítica en el desarrollo psicológico.

Es plausible especular que las mujeres tienden a evitar los conflictos con los padres para proteger la relacion de apego. Sabemos también que las chicas, en general, demuestran mayores niveles de implicación emocional y de ansiedad por la separación (Beyers y Goossens, 1999; Holmbeck y Wandrei, 1993; Lamborn y Steinberg, 1993) lo cual puede explicar su menor capacidad para conseguir autonomía. Distintas prácticas parentales en cuanto a los chicos y las chicas pueden explicar nuestros resultados: a las chicas se les concedían menos oportunidades para la autonomía, y estaban más restringidas; a los chicos se les socializa hacia mayor autonomía, y a las chicas a estar más vinculadas con los demás.

\section{Referencias}

Allen, J. P., Hauser, S. T., O’Connor, T. G., y Bell, K. L. (2002). Prediction of peer-rated adult hostility from autonomy struggles in adolescents' family interactions. Developmental Psychopathology, 14, 123-137.

Anderson, W. W., y Anderson, D. D. (1986). Thai Muslim adolescents' self, sexuality, and autonomy. Ethos, 14, 368-394.

Arnold, L. M. (2003). Gender differences in bipolar disorder. Psychiatric Clinics of 
North America, 26, 595-620.

Bartle, S. B., Anderson, S. A. y Sabatelli, R. M. (1988). A model of parenting style, adolescent individuation, and adolescent self-esteem: Preliminary findings. Journal of Adolescent Research, 4, 283-289.

Benaches, J. L. (1981). La autonomía como dimensión de la inserción social del adolescente de quince años. Psicologica, 2, 167-177.

Bergen, H. A., Martin, G., Richardson, A. S., Allison. S. y Roeger, L. (2003). Sexual abuse and suicidal behavior: a model constructed from a large community sample of adolescents. Journal of the American Academy of Child and Adolescence Psychiatry, 42, 1301-1309.

Beyers, W., y Goossens, L. (1999). Emotional autonomy, psychosocial adjustment and parenting: interactions, moderating and mediating effects. Journal of Adolescence, $22,753-769$.

Blos, P. (1967). The second individuation process of adolescence. Psychoanalytic Study of the Child, 22, 162-186.

Borresen, R. y Rosenvinge, J. H. (2003). Body dissatisfaction and dieting in 4,952 Norwegian children aged 11-15 years: less evidence for gender and age differences. Eat and Weight Disorders, 8, 238-241.

Bosma, H.A., Jackson, S.E., Zijsling, D.H. Cicognani, E., Xerri, M.L., Honess, T.M. y Charman, L. (1996). Who has the final say? Decisions on adolescent behaviour within the family. Journal of Adolescence, 19, 277-291.

Bumpus, M.F., Crouter, A.C., McHale, S.M. (2001). Parental autonomy granting during adolescence: exploring gender differences in context. Dev Psycol., 37, 163-173.

Chen, Z. Y. (1999). Ethnic similarities and differences in the association of emotional autonomy and adolescent outcomes: comparing Euro-American and Asian American adolescents. Psychology Report, 84, 501-506.

Chou, K. L. (2000). Emotional autonomy and depression among Chinese adolescents. Journal of Genetical Psychology, 161, 161-168.

Cohen, P., Kasen, S., Chen, H., Hartman, C., y Gordon, K (2003) Variations in patterns of developmental transitions in the emerging adulthood period. Developmental Psychology, 39, 657-669.

Dornbush, S. M., Erickson, K. G., Laird, J. y Wong, C. A. (2001). The relation of family and school attachment to adolescent deviance in diverse groups and communities. Journal of Adolescent Research, 16, 396-422. 
Douvan, E. y Adelson, J. (1966). The adolescent experience, New York: J. Wiley \& Sons.

Dowdy, B.B., Kliewer, W. (1998). Dating, parent-adolescent conflict, and behavioural autonomy. Journal of Youth and Adolescence, 4, 473-492.

Fleming, M. (1983). A separação adolescente-progenitores. Análise Psicológica, 4, 521542.

Fleming, M. (1992). O processso de separação-individuação do adolescente: contribuições da teoria psicanalítica. Revista Portuguesa de Psicanalise, 10/11, 89101.

Fleming, M., y Aguiar, A. I. (1992) A saída de casa e o processso de autonomia em jovens universitários e seus pais. Psicologia, 8, 329-337.

Fleming, M. (2005). Adolescent autonomy: desire, achievement and disobeying parents between early and late adolescence. Australian Journal of Educational and Psychology, 5, 1-16.

Frank, S. J., Schettini, A. M., y Lower, R. J. (2002). The role of separation-individuation experiences and personality in predicting externalizing and internalizing dimensions of functional impairment in a rural preadolescent and adolescent sample. Journal of Clinical Child and Adolescence Psychology, 31, 431-442.

Greenberger, E. (1984). Defining psycho-social maturity in adolescence. En P. Karoly, J.J. Steffen (Ed). Adolescent Behavior Disorders: Foundations and contemporary concerns, New York: Lexington Books.

Holmbeck, G.N. y Wandrei, M.L. (1993). Individual and relational predictors of adjustment in first-year college students. Journal of Counseling Psychology, 1, 7378.

Joseph, H. B., Reznik, I., Mester, R. (2003). Suicidal behavior of adolescent girls: profile and meaning. Israel Journal of Psychiatry and Related Sciences, 40, 209-219.

Josselson, R. (1980). Ego development in adolescence. En J. Adelson (Ed). Handbook of Adolescent Psychology, New York: Wiley.

Kiesner, J., Cadinu, M., Poulin, F. y Bucci, M. (2002). Group identification in early adolescence: its relation with peer adjustment and its moderator effect on peer influence. Child Development, 73, 196-208. 
Kiesner, J., Poulin, F., Nicotra, E. (2003). Peer relations across contexts: individualnetwork homophily and network inclusion in and after school. Child Development, 74, 1328-1243.

Konopka, G. (1983). Young girls: A portrait of adolescence. VIII. What is and what should be. Child \& Youth Services, 6, 157-171.

Lacombe, A.C., Gay, J. (1998). The role of gender in adolescent identity and intimacy decisions. Journal of Youth and Adolescence, 6, 795-802.

Lamborn, S.D., Steinberg, L. (1993). Emotional autonomy redux: revisiting Ryan and Lynch. Child Development, 64, 483-499.

Mahler, M.S., Pine, F. y Bergman, A. (1975). The psychological birth of the human infant, New York: Basic Books.

McElhaney, K. B., y Allen, J. P. (2001). Autonomy and adolescent social functioning: the moderating effect of risk. Child Development, 72, 220-235.

Meyer, R. (1988). Une approche des valeurs personnelles des adolescents. Enfance, 1, 75-86.

Moore, D. (1987). Parent-adolescent separation: the construction of adulthood by late adolescents. Developmental Psychology, 23, 298-307.

Noom, M. J., Dekovic, M., y Meeus, W. H. (1999). Autonomy, attachment and psychosocial adjustment during adolescence: a double-edged sword? Journal of Adolescence, 22, 771-783.

Noom, M. J., Dekovic, M., y Meeus, W. H. (2001). Conceptual analysis and measurement of adolescent autonomy. Journal of Youth and Adolescence, 5, 577595.

Peppitone, L.A. (1980). Adolescent separation: A developmental and intergenerational study of relationship. London: University Microfilms International.

Pinto, K.C. (2004). Intersections of gender and age in health care: adapting autonomy and confidentiality for the adolescent girl, Qual Health Res., 14 (1), 78-99.

Pinheiro, R. T., Sousa, P. L., Horta, B. L., Silva, R. A., Souza, R. M., y Fleming M. (2001). Cocaine addicts and their families: an empirical study of the process of identification. International Journal of Psychoanalysis, 82, 347-360.

Pipp, S., Shaver, P., Jennings, S., Lamborn, S. y Fischer, K.W. (1985). Adolescents' theories about the development of their relationships with parents. Journal of Personality and Social Psychology, 4, 991-1001. 
Purdie, N., Carrol, A. y Roche L. (2004) Parenting and adolescent self-regulation. Journal of Adolescence, 27, 663-676.

Ryan, R. M., y Lynch, J. H. (1989) Emotional autonomy versus detachment: revisiting the vicissitudes of adolescence and young adulthood. Child Development, 60, 340356.

Schmitz, M. F., y Baer, J. C. (2001). The vicissitudes of measurement: a confirmatory factor analysis of the Emotional Autonomy Scale. Child Development, 72, 207-219.

Silverberg, S.B. y Steinberg, L. (1987). Adolescent autonomy, parent-adolescent conflict and parental well-being. Journal of Youth and Adolescence, 3, 293-312.

Sloan, D. M. y Kornstein, S. G. (2003). Gender differences in depression and response to antidepressant treatment. Psychiatric Clinics of North America, 26, 581-594.

Smith, K. y Crawford, S (1986) Suicidal behavior among "normal" high school students. Suicide and Life Threatening Behaviors, 16, 313-325.

Steinberg, L. (1990). At the Threshold: the Developing Adolescent. En S. Feldman y G. Elliot, (Eds.), Cambridge, MA: Harvard University Press, pp. 255-276.

Steinberg, L. (1999). Adolescence. Boston: McGraw-Hill.

Steinberg, L., y Silverberg, S. B. (1986). The vicissitudes of autonomy in early adolescence. Child Development, 57, 841-851.

Stewart, A. J. y McDermott, C. (2004). Gender in psychology. Annual Review of Psychology, 55, 519-544.

Taradesh, A., Connolly, J., Pepler, D., Craig, W., y Costa, M. (2001) The interpersonal context of romantic autonomy in adolescence. Journal of Adolescence, 24, 365-377.

Verhofstadt-Deneve, L. M., Schittekatte, M. y Van Leeuwen, K. (2003). Gender differences in development pathways on self-evaluation from adolescence to adulthood: the Flanders longitudinal study. International Journal of Adolescence Medical Health, 15, 139-152.

Williamson, J.A. y Campbell, L.P. (1985). Parents and their children comment on adolescence. Adolescence, 79, 745-748.

Zwaanswijk, M., Verhaak, P. F., Bensing, J. M., van der Ende, J. y Verhulst, F. C. (2003). Help seeking for emotional and behavioural problems in children and adolescents: a review of recent literature. European Child and Adolescent Psychiatry, 12, 153-161. 